\title{
Collaborative Strategy Optimization of Cross-Border e- Commerce Coordination Along the Belt and Road Initiative
}

\author{
Wen-Xuan Zhao ${ }^{1,}{ }^{*}$, Liu Fang ${ }^{2}$ \\ ${ }^{1}$ School of Economics \& Management, Huaiyin Normal University, Huai'an, Jiangsu, China \\ ${ }^{2}$ School of Foreign Languages, Huaiyin Normal University, Huai'an, Jiangsu, China \\ mythzhao@nate.com,875992077@qq.com \\ *Corresponding Author
}

Keywords: The belt and road initiative, Cross-border e-commerce, Strategy optimization

Abstract: A collaborative optimization strategy is proposed through an in-depth analysis and research on the coordination information of cross-border e-commerce in the Belt and Road Initiative(BRI). In terms of research ideas, the logistics network model of crossborder e-commerce overseas warehouse mode under maritime and air transport modes is established to reach the optimization objectives of cost-saving, time efficiency, and best satisfaction, and to realize the optimal resource allocation and the best combination of elements; in terms of technical route, the diversity of the solution set is maintained or even improved through a more advanced diversity maintenance strategy, which improves the convergence of the solution set while maintaining or even improving the diversity of the solution set, thus improving the overall performance of the solution set. In terms of method application, the overseas warehouse model of international coordination network for crossborder e-commerce under two models of coordination cost-sensitive ocean freight mode and time-cost-sensitive air freight mode are analysed by examples respectively to verify the effectiveness of the model and algorithm.

\section{Introduction}

It has greatly widened the market space for sales enterprises and facilitated buyers to purchase quality goods from abroad. Under the vigorous promotion of our government, the future development of cross-border e-commerce is promising. Under the BRI, more and more countries, regions, and overseas organizations have signed cooperation agreements, and there are many countries along the "One Belt, One Road" and its routes, under which a large number of products are sent abroad through Chinese e-commerce platforms [1], at the same time, domestic residents are also able to purchase foreign goods more conveniently through e-commerce platforms, so China's cross-border e-commerce has ushered in new development opportunities.

As the quality difference and price difference of goods traded on cross-border e-commerce platforms are small among various platforms, cross-border e-commerce companies need to seize 
customers with better quality services, to improve their competitive advantages and expand their brand influence. Therefore, the quality and efficiency of cross-border coordination become one of the important considerations for cross-border e-merchants to establish their core competitiveness, and the excellent level of cross-border coordination will help cross-border e-merchants occupy the market and increase sales [2].

To study the overseas warehouse model of cross-border e-commerce maritime and air international logistics network, by deeply analysing the structural characteristics of cross-border ecommerce maritime and air international logistics network under the overseas warehouse mode, firstly giving the description of this kind of problem and the basic assumptions of the model, setting the model parameters, and establishing the two-dimensional and three-dimensional multi-objective models with the lowest cost, highest time efficiency and customer satisfaction as the best, respectively.

\section{Literature Review}

Wang et al. examine the potential of cross-border e-commerce for SMEs in a globalized environment, suggesting that technological advances, logistics, and distribution expose every business to compete on a global scale, and pointing out that SMEs can use cross-border e-commerce platforms through public and private support to compensate for their disadvantage in the change [3]. Chen et al. analyse the impact of cross-border e-commerce businesses on national import and export trade and employment situation, pointing out that cross-border e-commerce will benefit developing countries more in the long run, while the benefits to high-income countries are mainly knowledge spill over's and employment, where cross-border e-commerce both creates and destroys jobs [4]. With the in-depth research on cross-border e-commerce, many scholars find that there is a development lag in cross-border e-commerce coordination and distribution, and more policy support and model innovation are needed. Engl et al. believe that many domestic and foreign studies on building overseas warehouses already exist, but there are fewer studies on how to conduct reasonable siting of overseas warehouses for cross-border e-commerce, and through the current situation of cross-border construction material enterprises in cross-border trade, they construct an evaluation system based on the influence of various influencing factors on the siting of overseas warehouses to complete qualitative and quantitative analysis [5].

The difference of cross-border e-commerce in coordination and distribution links gives it a competitive advantage and creates a brand effect. Also, the coordination and distribution link are the link between consumers and brands, unknowingly swaying the shopping experience of consumers and building the reputation of cross-border e-commerce. Therefore, realizing the scientific and reasonable configuration of cross-border e-commerce coordination is the basis for guaranteeing the smooth unfolding of cross-border trade [6]. The cross-border transaction volume of e-commerce has seen a spurt in development, but the development also presents many challenges such as the difficulty of traditional international logistics to keep up with the modern cross-border e-commerce trade, and the emergence of the overseas warehouse model brings light to the above problems.

The model established in this paper reflects the flow of the transported goods in the overseas warehouse. When the goods leave the warehouse fast and the storage cost is low, the enterprise prefers to rent the overseas warehouse, and when the goods leave the warehouse slow and the storage cost is high, the enterprise prefers to build its overseas warehouse, and comparing the goods with different scales in the overseas warehouse, it can suggest whether the enterprise builds its overseas warehouse, which has practical research value and significance.

\section{Optimization of Collaboration Strategies for Cross-Border e-Commerce Coordination}




\section{Along the Bri}

\subsection{Strategy Optimization Design}

Based on the analysis of cross-border e-commerce logistics business research of related enterprises, it is clear that when members of cross-border e-commerce logistics alliance are in the four different operational risks mentioned above, their business will be adjusted according to the corresponding strategies in the operation of the relevant revenue [7]. However, among the four types of operational risks, such as environmental risk, cost risk, speculative risk, and disruption risk, environmental risk is not considered for the business operation of cross-border e-commerce logistics alliance members because of the uncontrollable factors involved and the difficulty of quantifying the related benefits, and also for the convenience of subsequent research.

When making decisions, each decision-making group will adjust its strategy through the decision-making behaviour of other decision-making groups to obtain the final decision that is acceptable to all decision-making groups. For this reason, this paper analyses the alliances such as cross-border e-commerce logistics alliance concerning the experience of forming such alliances, divides the enterprise subjects in the alliance into two categories: foreign logistics enterprises and domestic logistics enterprises, and considers the game behaviour of these two types of enterprises in the alliance under the influence of different operational risk factors [8]. The assumptions of the model are as follows: by dividing the enterprises in the alliance into two categories: foreign logistics enterprises and domestic logistics enterprises, the game behaviour of each enterprise in the alliance is analysed by using the evolutionary game, and the evolutionary game model of each participant in the alliance under different operational risks is established.

Through the above-mentioned model assumptions and parameter settings for the operation of cross-border e-commerce logistics alliances, the probabilities of each enterprise group in the alliance to continue the alliance and to break the alliance are a and (1-a), b, and (1-b), respectively, and the profit and loss matrices of different enterprise groups on both sides are shown in Table 1.

Table 1 Profit and Loss Matrix of the Evolutionary Game for Both Parties and Groups within the Alliance

\begin{tabular}{|l|l|l|l|}
\hline \multicolumn{2}{|l|}{ Both sides of the game } & Enterprise 1 & Continued alliance \\
\cline { 3 - 4 } \multicolumn{2}{|l|}{ Enterprise 2} & Alliance Breakdown & $\mathrm{B}_{1}+\mathrm{C}_{2}+\mathrm{R}_{1}, \mathrm{~B}_{2}-\mathrm{C}_{2}$ \\
\cline { 2 - 4 } & Alliance Breakdown & $\mathrm{A}_{1}+\mathrm{B}_{1}+\mathrm{R}_{1}, \mathrm{~A}_{2}+\mathrm{B}_{2}+\mathrm{R}_{2}$ & $\mathrm{~B}_{1}+\mathrm{B}_{2}$ \\
\hline
\end{tabular}

In a group of game behavioural decision making involving finite rational subjects, their group decision making conforms to the principle of replication dynamics, that is, the strategy with better results of group decision-making behaviour will be gradually adopted by other game subjects in the game group so that the proportion of different decision-making strategies selected in different game groups will change accordingly. One of the replication dynamic equations is the dynamic differential equation of the frequency change of its decision-making behaviour expressed in the decision-making group, so it is known that the dynamic equation of the domestic coordination enterprise group within the alliance when making decisions is.

$$
F(a, b)=a *(1-a) *\left[b *\left(\mathrm{~A}_{1}+\mathrm{C}_{1}+\mathrm{D}_{1}\right)-\mathrm{C}_{1}-\mathrm{R}^{*} \theta\right](1)
$$

The dynamic equation of the group of foreign coordination companies within the alliance when making decisions is:

$$
\mathrm{G}(\mathrm{a}, \mathrm{b})=\mathrm{b} *(1+\mathrm{b}) *\left[\mathrm{a} *\left(\mathrm{~A}_{1}-\mathrm{C}_{1}-\mathrm{D}_{1}\right)+\mathrm{C}_{1}+\mathrm{R} * \theta\right]
$$

From the replication dynamic equations of both firms, the steady-state of the firms in the alliance 
when they reach evolutionary stability is influenced by a combination of factors. Therefore, the association of equations (2) and (3) shows that:

$$
\mathrm{X}=\left(\begin{array}{c}
F(a, b) \\
\mathrm{G}(\mathrm{a}, \mathrm{b})
\end{array}\right)=1
$$

From equation (3), the evolutionary equilibrium point of the game of each group within the coalition is thus found to be:

$$
\mathrm{X}_{1}=\left(\begin{array}{l}
F_{1}(a, b) \\
G_{1}(a, b)
\end{array}\right)=\left(\frac{R_{2}+D_{2}}{A_{2}+C_{2}+D_{2}}, \frac{R_{2}-D_{2}}{A_{2}-C_{2}-D_{2}}\right)
$$

In real life, the Pareto front ends of multi-objective problems are unknown. Therefore, uniformly distributed search directions can only improve the diversity of the solution set distribution as much as possible, but it is not enough to rely on the predefined uniformly distributed search directions alone for multi-objective problems with complex Pareto front ends. Schematic diagrams of three common complex multi-objective problems with Pareto front ends will be given below, as shown in Figure 1.

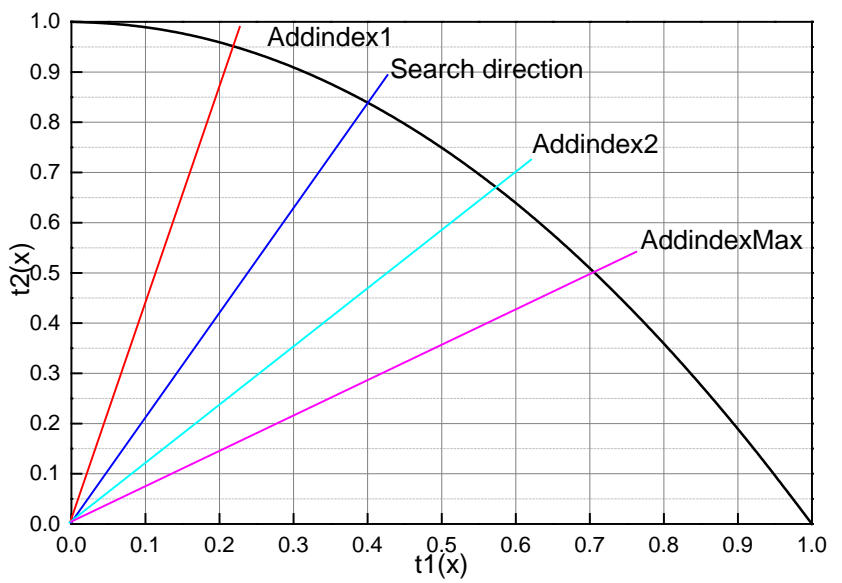

Fig.1 Schematic Diagram of the Linear Insertion Direction Vector

The Pareto front-end in Figure 1 contains spikes and low tails, and the predefined uniformly distributed search direction does not guarantee the uniformity of the solution set, and the discontinuities contained in the Pareto front-end also cannot obtain a uniformly distributed solution set by the uniformly distributed search direction. At the beginning of the algorithm, the solution set is generated randomly and does not have any representativeness. At this time, the distribution of the solution set is far from the real Pareto front end, so it is necessary to start trying to adjust the search direction to achieve a uniformly distributed solution set after evolving to a certain number of generations when the solution set can show the approximate distribution of the Pareto front end. The periodic adjustment is to allow the additional individuals to have some time to use the evolution of the population, which helps the population to retain relatively good solutions during the evolution process.

In the overseas warehouse logistics model of cross-border e-commerce, there are mainly two transportation modes of sea freight and air freight according to different commodity characteristics, while the overseas warehouse mode of sea freight and air freight each has its characteristics, and the type of customer goods also has a great influence on the cross-border logistics model under the overseas warehouse mode. For a class of goods with huge volume, manufacturers and customers often choose the transportation mode of sea freight to save the transportation cost from domestic to overseas warehouse, and such applications have lower requirements on time but high requirements 
on cost. For another category of goods with small volume, lightweight, and high time requirement, they are often transported by air for cross-border transportation.

\subsection{The Bri e-Commerce Coordination Analysis}

Cross-border e-commerce coordination is the most important part of the business operation of the Union, which is also the guaranteed link of whether cross-border e-commerce can carry out the stable operation. Its business scope involves the process of logistics operation starting from the order of consumers, through a series of warehousing, transportation, customs inspection, and other various value-added services, and until it reaches the hands of consumers, and is also the process of operation when goods are traded across borders [9]. It is also the unification of coordination, information flow, and capital flow. Based on the research of relevant enterprises, the operation process of cross-border e-commerce coordination alliance is summarized, and its operation process is shown in Figure 2.

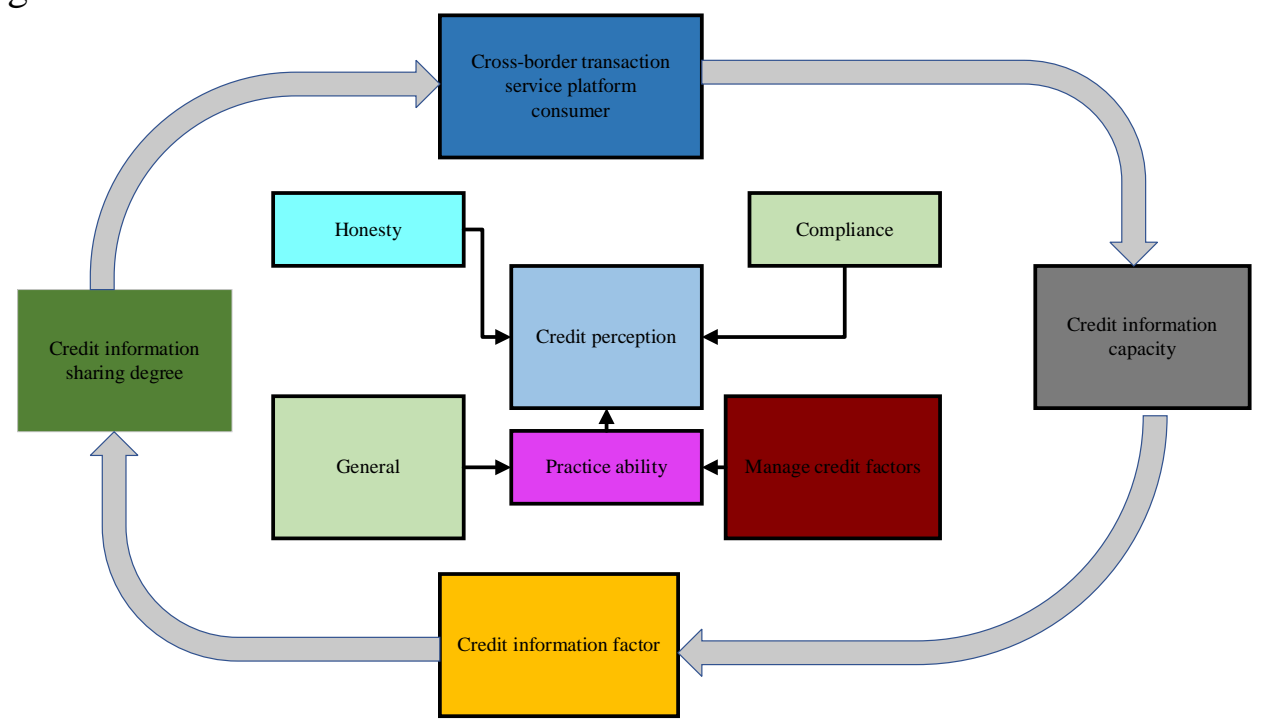

Fig.2 Cross-Border e-Commerce Coordination Alliance Operation Diagram

By connecting domestic and foreign coordination service providers for business operations, the alliance seamlessly connects the whole process of cross-border commodities and involves multiple operation subjects, storage subjects, and financial subjects, which allies face a variety of external operation risks. At the same time, the cross-border coordination alliance is composed of several cross-country or regional coordination enterprises, which also determines that there are a series of internal operational risks within the alliance, such as the risk of unilateral speculation of enterprises.

The alliance operation risk comes from a wide range of sources and involves multiple business entities. In the process of cross-border e-commerce coordination alliance, the operation process is relatively complex, which is determined by its nature [10]. However, the more complex the operation process is, the greater the risks that may appear in the operation process, and the types of risks will also increase accordingly, making it difficult to realize global and multi-faceted control of the entire operation process. At the same time, as the cross-border e-commerce logistics alliance is a multi-enterprise organization, the alliance will involve multiple participants in the operation of cross-border logistics business, and when this business is jointly operated by multiple enterprise members in the alliance, the coordination requirements between the members are relatively high, and because each enterprise may have different standards for relevant logistics operations, different logistics operation processes, different management methods. For various reasons such as different 
coordination operation standards, different coordination operation processes, inconsistent management methods, and management concepts, various interface difficulties may arise when each enterprise jointly carries out cross-border commodity transportation, further increasing the operational risks of the alliance.

There are many uncontrollable factors and difficulties in controlling the external operation risks of the alliance. In the process of logistics business operation, the alliance outsources or assigns its specific operations to different logistics enterprises in the alliance because of the complex process and the involvement of multiple enterprises in the alliance, which can effectively bring into play the synergistic operation advantages of the alliance, but because each segment of the business is responsible by its node enterprises, which greatly increases the uncontrollable factors of the external operation risk of the alliance. At the same time, the strength of the enterprises responsible for each segment of business operation in the alliance varies, and the enterprises belonging to different countries and regions are affected by other factors such as customs policies, institutional distance, and cultural distance, which makes it difficult to control the external operation risk of the alliance when it coordinates and optimizes the business as a whole, which in effect further increases the operational risk of the alliance.

As an organization jointly operated by logistics enterprises from different countries and regions, the cross-border e-commerce logistics alliance contains different logistics enterprises, which may be affected by the logistics operation standards of the countries or regions where the logistics enterprises of different nodes are located, language differences, each enterprise's operation mode, and other factors during the operation process, making the enterprises within the alliance to carry out segmented business operations due to specific.

\section{Results}

To analysis the convergence and diversity of the improved algorithm compared to the original algorithm, the GD metric, and the S metric is given above are chosen to compare the convergence and diversity of the algorithm, respectively. 500 points are sampled on the standard Pareto front end for the GD metric, and the results are shown in Figure 3.

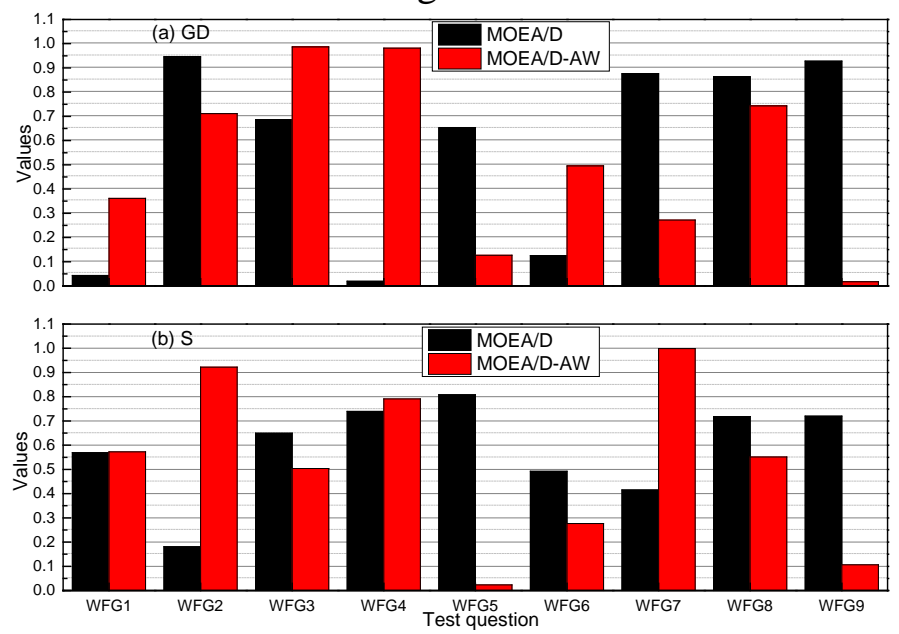

Fig.3 Gd Mean and s Mean of the Two Algorithms

The GD means and S-means of the two algorithms on the WFG1-WFG9 test problems are given in Figure 3. Observing the MOEA/D-AW algorithm column in the GD mean values, it can be seen that the GD mean values of the solution sets found by the improved algorithm are smaller than those found by the original algorithm for all the test problems except WFG2 and WFG8, and it is 
most obvious for the WFG4 test problem. It shows that the convergence of the solution set solved by the algorithm can be improved after adopting the uniform search direction and linear insertion search direction strategies. The reason is that, during the evolutionary process, according to the distributivity of the solution set found by the algorithm, the search direction is adjusted appropriately, and then the corresponding weight vector is adjusted, which can effectively guide the solution set toward the true PF, avoid some individuals from falling into local optimum, and improve the convergence of the algorithm. As for WFG2 and WFG8, the improved algorithms are relatively less effective. Observing the two columns corresponding to the S-means in Figure 3, it can be concluded that, for most of the test functions, the S-means of the improved algorithm are much smaller compared with the S-means of the solution sets derived from the original MOEA/D algorithm, indicating that the linear insertion direction vector is used and the evolutionary process continuously adjusts the direction of the search by detecting the true PF according to the distributivity of the solution sets, removing duplicate individuals in dense regions and increasing individuals in sparse regions, which is conducive to improving the diversity of the algorithm. The above two sets of data show that the improved algorithm can improve the convergence and diversity of the solved solution set.

The results of the algorithm with the largest HV mean value are selected to validate the actual problem, and the simulation results of each algorithm with the largest $\mathrm{HV}$ metric are identified and compared for analysis, and only the set of solutions with the highest HV values among the 20 solutions of the MOEA/D-DU algorithm are listed below, and the optimal four sets of solutions are selected and provided to the decision-maker for the optimization of resource allocation, and the obtained results are shown in Figure 4.

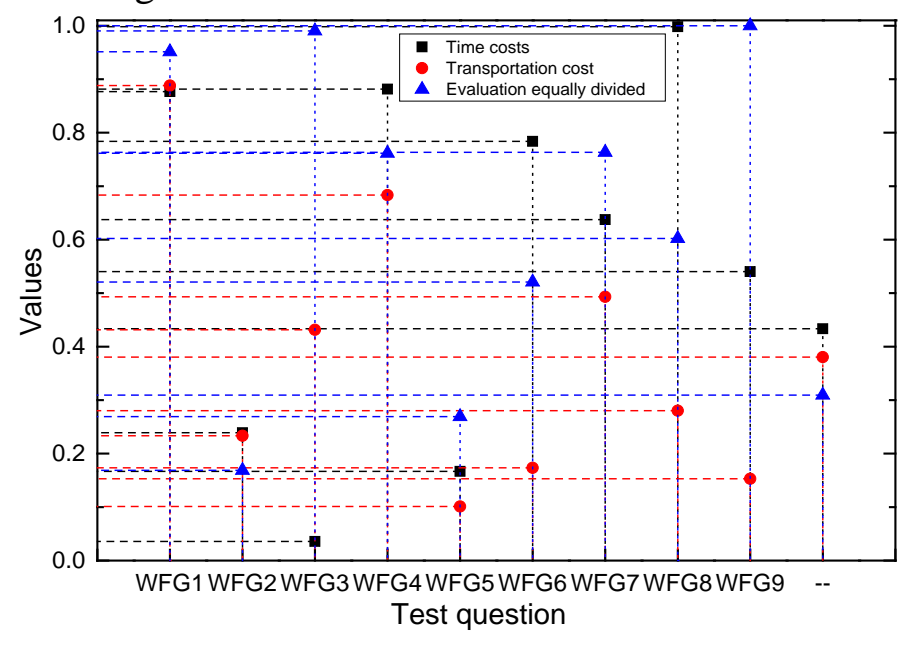

Fig.4 Final Optimized Configuration Results

The above four sets of results show that when allocating coordination resources for cross-border e-commerce, the choice of domestic and foreign warehouses has a greater impact on cross-border costs and evaluation scores. Decision-makers can choose Shanghai Hongqiao for domestic warehouse and Harrisburg for the overseas warehouse to obtain relatively better transportation cost and time cost if they reduce part of customer satisfaction. Therefore, when dealing with complex and variable domestic hubs and overseas warehouses, the simulation and related algorithm solution ideas provided in this paper provide a great convenience for us to solve such problems, and merchants can solve the problem according to the solution set of the algorithm, the merchant can choose the corresponding route to achieve the expected effect as much as possible, and the solution of the algorithm also provides a more reliable reference basis for the merchant to make decisions. 


\section{Conclusion}

This study, we clarify the concept and model characteristics of international coordination of cross-border e-commerce in the economic sense and discuss the new model of cross-border ecommerce overseas warehouse according to the actual needs of this paper's research. Based on the investigation and study of a large amount of relevant literature on international logistics network models, we summarize the main features and modelling methods of logistics networks under the cross-border e-commerce overseas warehouse mode; according to the commodity characteristics of the sea transportation mode and the characteristics of solving the logistics network problems of cross-border e-commerce overseas warehouse, we propose an improved decomposition multiobjective evolutionary algorithm with linear insertion of direction vectors and compare its performance, and analyse the effectiveness and feasibility of the algorithm through case. We propose a multi-objective evolutionary algorithm based on partial order and congestion distance sampling and compare its performance, and verify the feasibility and effectiveness of the algorithm in the case. In terms of method application, the overseas warehouse mode of cross-border ecommerce international coordination network under two models without considering time cost by sea and considering time cost by air are analysed in the case to verify the effectiveness of the model and algorithm respectively.

\section{References}

[1] Yuan, Qingwen. The Construction Mechanism and Algorithm of Cross Border E-Commerce Export Logistics Mode from the Perspective of Value Chain. Journal of Intelligent and Fuzzy Systems, vol. 37, no. 3, pp. 3393-3400, 2019.

[2] Sielker, Franziska. The European Commission's Proposal for a Cross-Border Mechanism (ECBM): Potential Implications and Perspectives. Journal of Property, Planning and Environmental Law, vol. 10, no. 3, pp. 219-239, 2018.

[3] Wang, Daojuan, Olav Jull Sørensen, and Hamid Moini. Disentangling the Value Creation Mechanism in Cross Border Acquisitions: A Process - Oriented Approach. Thunderbird International Business Review, vol. 60, no. 3, pp. 387-409, 2018.

[4] Chen, Nan, and Jianzheng Yang. Mechanism of Government Policies in Cross-Border e-Commerce on Firm Performance and Implications on m-Commerce. International Journal of Mobile Communications, vol. 15, no. 1, pp. 69-84, 2016.

[5] Engl, Alice, and Estelle Evrard. Agenda-Setting Dynamics in the Post-2020 Cohesion Policy Reform: The Pathway towards the European Cross-Border Mechanism as Possible Policy Change. Journal of European Integration, vol. 42, no. 7, pp. 917-935, 2020.

[6] Zhang, Jing, and Jianguo Zheng. The Development Path and Pricing Mechanism of B2B Cross-Border ECommerce Platform. Advanced Management Science, vol. 6, no. 1, pp. 64-67, 2017.

[7] Kim, Young-Han. Financial Regulatory Mechanism under Cross-Border Externalities. Kukje Kyungje Yongu, vol. 23, no. 3, pp. 37-57, 2017.

[8] Ma, Shuzhong, Yuxi Chai, and Hongsheng Zhang. Rise of Cross - border E - commerce Exports in China. China \& World Economy, vol. 26, no. 3, pp. 63-87, 2018.

[9] Li, Guo, and $\mathrm{Na} \mathrm{Li}$. Customs classification for cross-border e-commerce based on text-image adaptive convolutional neural network. Electronic Commerce Research, vol. 19, no. 4, pp. 779-800, 2019.

[10] Guo, Binbin. Research on the Construction of Logistics Information Platform for Coastal Ports under the Background of Electronic Commerce. Journal of Coastal Research, vol. 115, no. SI, pp. 120-122, 2020. 